The solid lines in Fig. 1 are least squares fits of the CDAD data to the summation $\Sigma_{L} a_{L} P_{L}^{1}(\cos \theta)$, which describes CDAD distributions taken with the experimental arrangement used in this work. ${ }^{3,4}$ The $P_{L}^{1}(\cos \theta)$ are associated Legendre polynomials of order $L(M=1)$ and $\theta$ is the angle between the linear polarization and electron propagation vectors. Symmetry considerations restrict $L$ to even values in the range $2 \leqslant L \leqslant 2 J$, where $J$ is the smaller of the two quantities: the total angular momentum of the probed state or the number of linearly polarized pump photons. The expansions coefficients $a_{L}$ are actually products of two other parameters, $A^{(L)}$ and $\bar{\beta}_{L}$, which describe the alignment of the excited state and the ionization dynamics of the boundcontinuum transition, respectively. ${ }^{4}$ For the spin-unresolved $R_{21}+S_{11}(1 / 2)$ rotational branch line $(J=3 / 22(R), 5 /$ $2(S), N=2),{ }^{6}$ the CDAD data was found to be well described by only the first term in the expansion, i.e., $a_{2} P_{2}^{1}$ $(\cos \theta)$ [see Fig. 1(a)]. In contrast, the $S_{21}(1 / 2)$ branch line $(J=5 / 2, N=3)$ required the full $a_{2} P_{2}^{1}+a_{4} P_{4}^{1}$ expansion to adequately fit the data with $a_{4} / a_{2}=-0.08(2)$. Inclusion of the $L=4$ term is clearly visible in Fig. 1(b) as the fitted CDAD distribution is no longer symmetric about $\pm \pi / 4$.

Whereas the fit of the experimental $R_{21}+S_{11}(1 / 2)$ CDAD data indicated a very small $L=4$ component, the calculations of Dubs et al. ${ }^{3}$ (shown as dashed lines in Fig. 1) result in a $a_{4} / a_{2}$ ratio of -0.12 which breaks the $\pm \pi / 4$ symmetry of the CDAD curve. For the $S_{21}(1 / 2)$ branch line [Fig. 1(b) ], the calculations predict a larger $L=4$ contribution of the CDAD curve $\left(a_{4} / a_{2}\right)=-0.27$ than that extracted from the experimental fit, however, the overall shift of the CDAD intensity maximum towards $\pm \pi / 2$ is clearly reproduced by experiment. Furthermore, the increase in the
$L=4$ component in going from the $R_{21}+S_{11}(1 / 2)$ to the $S_{21}(1 / 2)$ branch line is consistent with the observed CDAD curves. It should be emphasized that the normalization used to plot the CDAD curves in Fig. 1, i.e., $I_{\mathrm{CDAD}} / I_{L, R}\left(\theta=0^{\circ}\right)$, places the comparison of the calculations and experiment on a quantitative basis. The overall good agreement between the theoretical and experimental CDAD amplitudes suggest that the bound-free ionization dynamics are realistically treated in the theoretical model of Dubs and co-workers. Experimental improvements in laser beam overlap and shotto-shot power stabilities should increase the statistical quality of these measurements and thereby permit a more detailed comparison of the shapes of the CDAD distributions with theoretical predictions.

The authors are indebted to Dr. Sham N. Dixit, Richard L. Dubs, and Professor Vincent McKoy for helpful discussions and for providing details of their calculations. This work was performed at Brookhaven National Laboratory and was supported by the Department of Energy, Office of Basic Energy Sciences, Division of Chemical Physics under Contract No. DE-AC02-76CH00016.

\footnotetext{
a) Support provided by the Donors of the Petrolem Fund, administered by the American Chemical Society.

'R. L. Dubs, S. N. Dixit, and V. McKoy, Phys. Rev. Lett. 54, 1249 (1985).

${ }^{2}$ R. L. Dubs, S. N. Dixit, and V. McKoy, Phys. Rev. B 32, 8389 (1985).

${ }^{3}$ R. L. Dubs, S. N. Dixit, and V. McKoy, J. Chem. Phys. 85, 656 (1986).

${ }^{4}$ R. L. Dubs, S. N. Dixit, and V. McKoy, J. Chem. Phys. (to be published).

${ }^{5}$ J. R. Appling and M. G. White (to be published); see also W. A. Chupka, A. M. Woodward, S. D. Colson, and M. G. White, J. Chem. Phys. 82, 4880 (1985).

'The $A^{2} \Sigma^{+}, v=0$ level is well described by Hund's case (b) coupling in which $J=N \pm 1 / 2$ where $N$ is the rotational quantum number. The spindoublets cannot be resolved with the present laser system.
}

\title{
Reaction kinetics on clusters and islands ${ }^{\text {a) }}$
}

\author{
J.S. Newhouse and R. Kopelman \\ Department of Chemistry, University of Michigan, Ann Arbor, Michigan 48109
}

(Received 2 July 1986; accepted 29 September 1986)

Our Monte Carlo simulations yield anomalously high reaction orders ( $X=20$ or higher) for elementary binary reactions on finite percolation clusters and monodisperse island ensembles. We find good agreement with exciton fusion reactions on naphthalene percolation clusters in isotopic mixed crystals. This has important implications for verylow-dimensional (zero to unity) heterogeneous kinetics.

The elementary binary reaction $\mathbf{A}+\mathbf{A} \rightarrow$ Products has been of much interest recently ${ }^{1-10}$ in connection with the problems of heterogeneous chemical kinetics. For instance, ${ }^{10-13}$ a nonclassical behavior has been demonstrated for the reaction order $X$, defined by

$$
R=K[\mathrm{~A}]^{X},
$$

where $R$ is the steady-state reaction rate, [A] the steadystate reactant concentration, and $K$ the rate constant. Specifically, instead of the classical result of $X=2$, values of $2.45,2.5$, and 3 have been obtained for the Sierpinski gasket, the critical percolation cluster and the one-dimensional lattice, respectively. ${ }^{11-13}$ This problem is of direct interest to chemical reactions and to exciton fusion (annihilation), ${ }^{1-13}$ and of indirect interest to radiation damage in semiconductors, electron-hole recombination, biological population studies, and matter-antimatter distribution in the universe. ${ }^{14}$ In fact, four years $\mathbf{a g o}^{5}$ it was claimed that triplet exciton fusion in isotopic mixed naphthalene crystals showed $X$ values up to 20 or higher for a certain alloy concentration range. This was interpreted in terms of guest clus- 
ters, below the critical percolation concentration. Recently a theoretical relation has been given ${ }^{11,13}$ for the steady-state reaction order: $X=1+2 / d_{s}$ for $d_{s}<2$, where $d_{s}$ is the spectral dimension. This gives $X=3$ for a linear lattice $\left(d_{s}=1\right)$, and for $d_{s}<1$ obviously $X>3$. In principle, for a finely divided reaction space, whether "fractal dust"15 or Euclidean dust, one expects $d_{s} \rightarrow 0$ and thus $X \rightarrow \infty$. It thus seemed to be of both experimental and theoretical interest to study this problem for a disjoint topology. We concentrated on finite percolation clusters because of their relation to the experimental system. ${ }^{5}$

The simulation method is that of Ref. 12 (and references therein). Percolation clusters are formed via the Hoshen algorithm ${ }^{16}$ on a square lattice of size $200 \times 200$. Typically, 10 configurations were formed for each guest concentration (C). Random walkers land at random on all guest sites with an overall rate $R$ (one walker per $1,2,4,8,16$, or 32 computer time steps). The walkers move at random on all the percolation clusters ${ }^{17}$ and when they collide they annihilate ${ }^{7,12}$ $(A+A \rightarrow 0)$. This procedure is repeated for each of the run's 150000 or more steps. Long before reaching the last 50000 steps, a steady-state walker population has been attained. At least four runs were performed for each point. Similar simulations were performed on monodisperse island ensembles. A system of $10895 \times 5$ square islands was populated $(R=2,4,8,16)$ as well as one with $32410 \times 10$ islands.

The rate exponent $(X)$ is derived from the slope of log (new walkers added per 1000 steps) vs log (average population of walkers over last 50000 steps of each of four runs), following Eq. (1). A least-squares analysis of the percolation cluster results gives the curve in Fig. 1. The reduced concentrations $\left(C / C_{c}\right)$ for this curve are based on ${ }^{18}$ $C_{c}=0.593$. For the $5 \times 5$ island ensemble we get $X \cong 15$ and for the $10 \times 10$ ensemble $X \cong 5$. The simulations were performed on a Charles River Data Universe 68000 superminicomputer and on a CRAY XMP-48 Supercomputer (Cray Research Inc., Mendota Heights, $\mathrm{MN}$ ).

The experimental values for exciton annihilation were

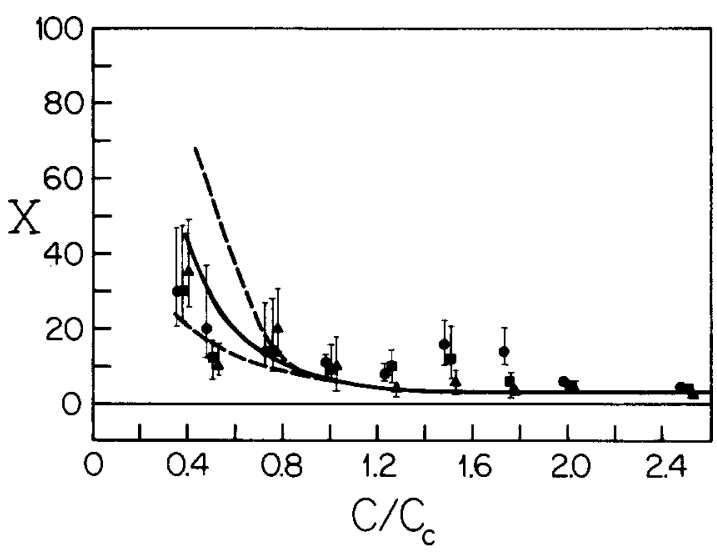

FIG. 1. (a) Simulation results (line) for the reaction order $X$ vs the reduced guest concentration $\left(C / C_{c}\right) . C_{c}=0.593$. The dashed lines give rough error bounds. (b) Experimental results for the reaction order $X$ vs the reduced guest concentration $\left(C / C_{c}\right)$. Based on exciton annihilation (Ref. 5 ) in naphthalene/naphthalene- $d_{8}$ mixed crystals at $2 \mathrm{~K}$. Relative quasi-steadystate exciton densities: 1 (triangles), 0.1 (squares), 0.01 (circles). $C_{c}=0.08$ (see the text). taken from the isotopically mixed crystal investigation of P. W. Klymko. ${ }^{5}$ We note that the effective triplet exciton percolation concentration $\left(C_{c}\right)$ is about $8 \%$ (roughly fourth-nearest-neighbor percolation problem ${ }^{20}$ ). However, this effective $C_{c}$ for the dynamic percolation concentration ${ }^{21}$ is somewhat dependent on the exciton density (and on the time scale). Thus the reduced $\left(C / C_{c}\right)$ experimental concentrations involve a large uncertainty (which is not so for the absolute concentrations $C$ given in Ref. 5). This is particularly true for the low exciton density points. For comparison with the simulations, the experimental results had to be plotted on a reduced concentration scale. However, only the "triangles" (high exciton-density points) should be compared quantitatively with the simulation curve.

Figure 1 shows that well above the critical percolation concentration $\left(C / C_{c}>1\right)$ one obtains the classical result $X=2$ for the binary reaction order (a possible logarithmic correction $^{22}$ for a two-dimensional reaction is within our experimental uncertainties, but is under separate investigation). At $C / C_{c} \approx 1$ the result is $X \cong 4$. This is obviously higher than the value of $X=2.5$ obtained $^{12}$ when the reacting walkers were limited to the largest ("percolating") cluster only, i.e., when the finite clusters were not populated. The effects of finite clusters (at $C_{c}$ ) and the "crossover" effect (from fractal to classical, for $C / C_{c}>1$ ) were studied earlier for the problem of the single random walker, ${ }^{23,24}$ and the crossover effects were confirmed for random walker reactions. ${ }^{25}$ Here our emphasis is on the behavior for finite clusters $\left(C / C_{c}<1\right)$, a regime that has not yet been studied, and that cannot be studied well via single random walks. This regime is now found to give the largest deviations from classical kinetics.

Experimentally, the regime below the critical concentration, i.e., the regime of finite clusters (or islands), is easily accessible. However, the very large exponents $(X)$ necessarily imply larger uncertainties (for the same uncertainties in reaction rate and population). The overall agreement between experiment and simulation is quite satisfactory, especially considering the fact that this is the first quantitative rationalization of the experimental data.

In summary, our Monte Carlo simulations on disjoint percolation clusters and on monodisperse island ensembles give steady-state binary reaction orders well above the classical value of 2 , with effective values higher by more than an order of magnitude. This is in excellent agreement with previously unexplained exciton annihilation experiments. The hitherto only suspected very large anomalies for very-lowdimensional heterogeneous kinetics (effective dimension between zero and unity) are confirmed. A new approach to heterogeneous (low dimensional) kinetics may be required, radically different from the traditional concepts of homogeneous (three-dimensional) chemical kinetics. The subone-dimensional world of reaction kinetics may be as rich as that between one and three dimensions.

We thank Cray Research Inc. (Mendota Heights, MN) for time on their XMP-48 supercomputer.

\footnotetext{
a) Supported by NSF Grant No. DMR 8303919.

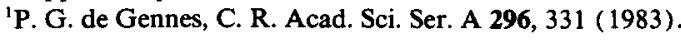


${ }^{2}$ P. Evesque and J. Duran, J. Chem. Phys. 80, 3016 (1984).

${ }^{3}$ J. Klafter, A. Blumen, and G. Zumofen, J. Stat. Phys. 36, 561 (1984).

${ }^{4}$ C. L. Yang, P. Evesque, and M. A. El-Sayed, J. Phys. Chem. 89, 3442 (1985).

${ }^{5}$ P. W. Klymko and R. Kopelman, J. Phys. Chem. 86, 3686 (1982).

${ }^{6}$ P. W. Klymko and R. Kopelman, J. Phys. Chem. 87, 4565 (1983).

${ }^{7}$ R. Kopelman, J. Hoshen, J. S. Newhouse, and P. Argyrakis, J. Stat. Phys. 30, 355 (1983).

${ }^{8} \mathrm{~K}$. Kang and S. Redner, Phys. Rev. A 32, 435 (1985).

${ }^{9}$ R. Kopelman, S. Parus, and J. Prasad, Phys. Rev. Lett. 56, 1742 (1986).

${ }^{10}$ R. Kopelman, J. Stat. Phys. 42, 185 (1986).

${ }^{11}$ L. W. Anacker and R. Kopelman, J. Chem. Phys. 81, 6402 (1984).

${ }^{12}$ J. S. Newhouse and R. Kopelman, Phys. Rev. B 31, 1677 (1985).

${ }^{13}$ L. W. Anacker, R. P. Parson, and R. Kopelman, J. Phys. Chem. 89, 4758 (1985).

${ }^{14}$ L. W. Anacker and R. Kopelman, Phys. Rev. Lett. (in press).

${ }^{15}$ B. B. Mandelbrot, The Fractal Geometry of Nature (Freeman, San Fran- cisco, 1983).

${ }^{16} \mathrm{~J}$. Hoshen and R. Kopelman, Phys. Rev. B 14, 3438 (1976).

${ }^{17}$ J. Hoshen and R. Kopelman, J. Chem. Phys. 65, 2817 (1976).

${ }^{18} \mathrm{~J}$. Hoshen, R. Kopelman, and J. Newhouse, J. Phys. Chem. (in press).

${ }^{19}$ P. W. Klymko, Ph.D. thesis, University of Michigan, Ann Arbor (1984).

${ }^{20}$ J. Hoshen, R. Kopelman, and E. M. Monberg, J. Stat. Phys. 19, 219 (1978).

${ }^{21}$ A. H. Francis and R. Kopelman, Laser Spectroscopy of Solids, Second Edition, Topics in Applied Physics, edited by W. M. Yen and P. M. Selzer (Springer, Berlin, 1986), Vol. 49, p. 241.

${ }^{22}$ D. C. Torney and H. M. McConnell, Proc. R. Soc. Lond. Ser. A 387, 147 (1983).

${ }^{23}$ P. Argyrakis and R. Kopelman, Phys. Rev. B 29, 511 (1984).

${ }^{24}$ P. Argyrakis and R. Kopelman, J. Chem. Phys. 81, 1015 (1984); 83, 3099 (1985).

${ }^{25}$ J. S. Newhouse, p. Argyrakis, and R. Kopelman, Chem. Phys. Lett. 107, 48 (1984).

\title{
Ion dip spectroscopy of higher excited vibronic states of aniline
}

\author{
Jack A. Syage ${ }^{\text {a) }}$ and John E. Wessel ${ }^{\text {b) }}$ \\ The Aerospace Corporation, P.O. Box 92957, Los Angeles, California 90009
}

(Received 2 September 1986; accepted 1 October 1986)

Double resonant ion dip spectroscopy (IDS) ${ }^{1-4}$ via single vibronic level (SVL) excitation in a supersonic molecular beam ${ }^{3}$ provides a powerful means for detecting higher excited electronic states and their underlying vibrational structure. ${ }^{2}$ These excited states are often too short-lived to be detected by conventional ionization or fluorescence detection methods. Direct detection by multiphoton absorption is likewise difficult to implement due to the low optical densities for such transitions in molecular beams.

Using two-color tunable excitation in a supersonic molecular beam, time-of-fight (TOF) mass spectrometer, we have recorded SVL absorption spectra corresponding to absorption from the first excited single state $\left(S_{1}\right)$ origin to a higher excited electronic state $\left(S_{n}\right)$ of aniline and aniline- $d_{5}$. The aniline $S_{n}$ spectrum in Fig. 1 was recorded by generating an ion signal by resonance two-photon ionization (R2PI) via the $S_{1}\left(\widetilde{A}^{1} B_{2}\right)-S_{0}\left(\widetilde{X}^{1} A_{1}\right) 0_{0}^{\circ}$ transition $\left(\omega_{1}\right)$ while a second dye laser $\left(\omega_{2}\right)$, overlapped and synchronous in time, was tuned through the $S_{n}(v) \leftarrow S_{1}(0)$ resonances. These absorptions compete with ionization, thereby creating the ion dip signals. The experimental apparatus will be described in detail elsewhere. ${ }^{5}$ Briefly, two tunable dye lasers are used to generate $\omega_{1}$ and $\omega_{2}$. The differentially pumped molecular beam apparatus employed a pulsed supersonic nozzle 6 which was operated at $10 \mathrm{~Hz}, 50^{\circ} \mathrm{C}, 50 \mathrm{psi} \mathrm{He}$, with a $500 \mu \mathrm{m}$ aperture. Excitation spectra were mass selected by gating the appropriate TOF ion signal (in the present case, the parent ion, $\mathbf{M}^{+}$).

The identification of the ion dip spectra as belonging to an $S_{n} \leftarrow S_{1}$ absorption was made by ruling out other possible mechanisms, namely down-pumping to vibrational levels in $S_{0}$ or absorption by parent ion, $\mathrm{M}^{+}$, to give fragments [ $\mathrm{M}-$ $\mathrm{m}]^{+}+\mathrm{m}$. The first possibility (also referred to as stimulat- ed emission pumping) can be ruled out by knowledge of the $S_{0}$ vibrational levels and transition moments. The possibility that $\mathbf{M}^{+}$absorbs light to give fragment ions, thereby depleting the $\mathrm{M}^{+}$ion signal, has also been ruled out by recording ion dip spectra for total ion signal. Furthermore, by delaying the $\omega_{2}$ pulse by $8 \mathrm{~ns}$, the ion dip spectrum disappeared. Since $\mathrm{M}^{+}$ion depletion by dissociative absorption should not be very sensitive to delay in the absorbing light, our result is consistent only with an $\omega_{2}$ absorption that competes with ionization from the $S_{1}$ electronic state.

The $0_{0}^{0}$ transition wavelengths for $S_{n} \leftarrow S_{1}$ occur at 770.9 $\mathrm{nm}$ (aniline) and $779.2 \mathrm{~nm}$ (aniline- $d_{5}$ ) which correspond to absolute energies above the $S_{0}$ vibrationless level of 47012 $\mathrm{cm}^{-1}(5.829 \mathrm{eV})$ and $47045 \mathrm{~cm}^{-1}(5.833 \mathrm{eV})$, respectively. The $S_{n}$ vibrational intervals identified in Fig. 1 compare reasonably well with previously observed $S_{0}$ and $S_{1}$ vibrations, $^{7}$ indicating that the $S_{n}$ state is characterized by geometry and force constants similar to the lower energy electronic states.

Recent studies on the MPI of aniline ${ }^{8-10}$ have revealed a strong visible region absorption that occurs from the $S_{1}$ level and which competes effectively with ionization. These studies involved $532 \mathrm{~nm}$ absorption in a supersonic molecular beam, ${ }^{8} 532 \mathrm{~nm}$ absorption in a thermal vapor cell,' and fixed wavelength visible absorption in a thermal vapor cell, ${ }^{9}$ and fixed wavelength visible absorption in an effusive molecular beam. ${ }^{10}$ The excitation energies in these studies were as much as $6000 \mathrm{~cm}^{-1}$ greater than those used in the present work and hence one cannot assume that the same excited state is being monitored. Moreover, since these results did not involve SVL excitation using tunable light, no determination of the electronic state energy or vibrational frequencies was made. We therefore refer to this higher energy state as $S_{n^{\prime}}$. Since, the apparent $S_{n^{\prime}}$ state did not appear to undergo 